\title{
Three-dimensional atom mapping of dopants in Si nanostructures
}

\author{
Keith Thompson ${ }^{\text {a) }}$ \\ Imago Scientific Instruments, 6300 Enterprise Lane Suite 100, Madison, Wisconsin 53719 \\ John H. Booske \\ Department of Electrical Engineering, University of Wisconsin, Madison Wisconsin 53703 \\ David J. Larson and Thomas F. Kelly \\ Imago Scientific Instruments, 6300 Enterprise Lane Suite 100, Madison, Wisconsin 53719
}

(Received 23 March 2005; accepted 14 June 2005; published online 27 July 2005)

\begin{abstract}
Atom-probe tomography has successfully mapped three-dimensional (3D) dopant atom distributions in nanoscale volumes of $\mathrm{Si}$ subjected to various processing procedures. The $3 \mathrm{D}$ evolution of dopants, specifically effects such as dopant clustering and grain-boundary segregation, were analyzed in implanted polycrystalline Si gate contacts and implanted shallow junctions. A cluster of dimensions $2 \times 7 \times 8 \mathrm{~nm}^{3}$ and containing $264 \mathrm{~B}$ atoms, was identified at the intersection of three poly-Si grains, verifying that annealing highly overdoped thin poly-Si layers does not facilitate uniformly doped and highly conductive gate contact layers. (C) 2005 American Institute of Physics. [DOI: 10.1063/1.2005368]
\end{abstract}

The downsizing of $\mathrm{Si}$ devices continues to drive advances in the electronics industry. Next generation nanodevices require novel diagnostic techniques in order to monitor the effects of macroprocessing on the nanoscale microstructure, to correlate the discovery of new nanoscale phenomena to macrorecorded events, and to understand how local nonuniformities of atomic distributions relate to electrical performance. It is increasingly apparent that this requires a three-dimensional (3D) microscopy technique that provides the location and elemental identity of atoms within a nanostructure. A practical 3D atomic mapping instrument should accurately measure, analyze, and display the composition of volumes of semiconducting materials that range in scale from 1 to $100 \mathrm{~nm}$.

An area of specific interest is the mapping of dopant atoms in Si. Great strides have been made to meet these needs via transmission electron microscopy (TEM) (Refs. 1 and 2). While TEM provides critical information regarding the physical composition of nanostructures, the most advanced electron microscopes have difficulty providing the volumetric dopant mapping desired by the semiconductor community. Alternatively, nano-secondory-ion-mass spectorocopy(sims) instruments using liquid metal ion sources may provide $\sim 50 \mathrm{~nm}$ lateral resolution, but this is still too coarse for quantitative analysis of nanostructures. ${ }^{3}$

A combination of recent innovations in instrumentation technology and specimen preparation-primarily enabled by the use of a "local electrode"-has transformed the traditional atom probe into a tool that provides such a capability. ${ }^{4,5}$ Recent experiments demonstrate that atom probing with a local electrode provides a $\sim 100 \mathrm{~nm}$-diam field of view and collection rates of $1 \mathrm{~nm} / \mathrm{min}$ for a $100-\mathrm{nm}-$ diam analysis region. This article describes several new experimental measurements that illustrate the powerful capabilities of this new nanoimaging technique in monitoring dopant distributions in shallow implanted wafers and in implanted poly-Si wafers which contain significant grain structure.
Samples for analysis are formed into atom-probe specimens by etching an array of tall $(>80 \mu \mathrm{m}), \sim 5 \mu \mathrm{m}$-diam posts out of the Si surface, (Fig. 1). The posts are sharpened with a focused ion beam (FIB) to a radius of curvature at the apex of 50-100 nm Ref. 6. A capping material such as Pt, $\mathrm{Au}$, or $\mathrm{Ni}$ is deposited on the specimen surface to protect it from Ga damage during the sharpening process. The capping layer may be left in place during analysis or removed prior to analysis via field ion milling. If the cap is not removed by field ion milling, it is removed as part of the analysis, creating an atomic map of both the cap material and the Gadamaged regions within the cap. This enables one to analyze the extent of $\mathrm{Ga}$ penetration to ensure that the region of interest, e.g., the Si surface, is not affected by the specimen preparation process.

The effectiveness of atom-probe microscopy at characterizing ultrashallow junctions was evaluated by analyzing a $6-\mathrm{keV}, 10^{15} / \mathrm{cm}^{2}$ As implant in Si. The sample was annealed at $1050{ }^{\circ} \mathrm{C}$ for $30 \mathrm{~s}$ in a $\mathrm{N}_{2}$ ambient whose background oxygen concentration was maintained at 100 ppm. Figure 2 displays a 3D map of As implanted dopants in Si. The As atoms are shown as large spheres and the $\mathrm{Si}$ atoms as points. To improve visual clarity, only $0.2 \%$ of the $\mathrm{Si}$ atoms are shown. The region selected for analysis is a cylinder $50 \mathrm{~nm}$ in diameter and $200 \mathrm{~nm}$ in depth. A one-dimentional(1D) compositional profile was created by slicing the 3D atom probe data into 1 -nm sections along the $z$ direction. For comparison, a PHI quadrupole SIMS $\left(5 \mathrm{keVCs}^{+}\right)$analysis on an adjacent region of the sample was performed. Figure 3 displays the comparative profiles. The noise floor for this particular atom-

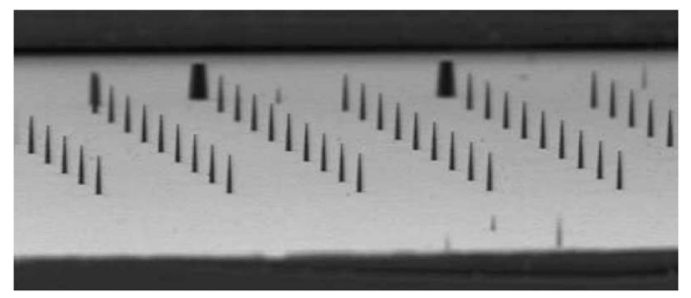

FIG. 1. SEM image of a Si microtip coupon.

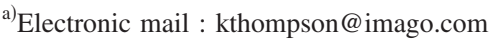




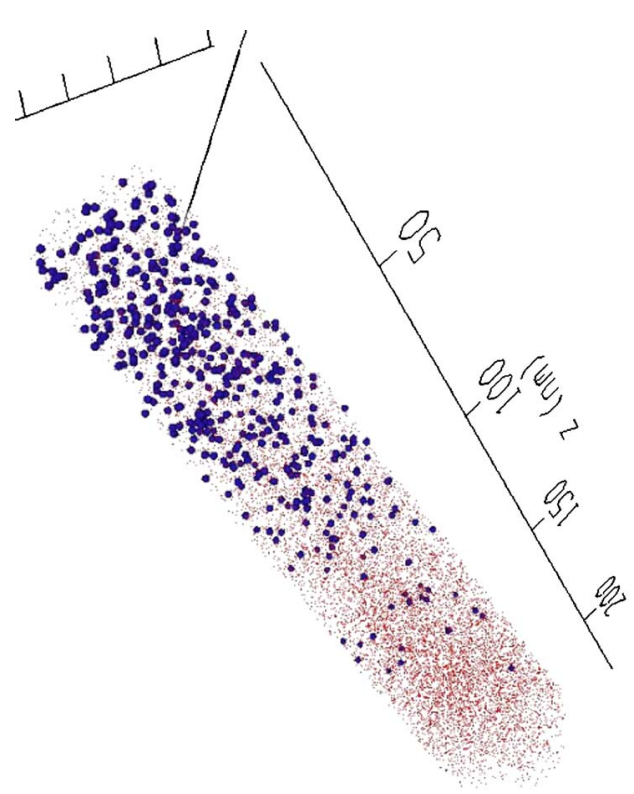

FIG. 2. (Color online) An atomic map of As (large spheres) implanted into $\mathrm{Si}$ (red dots). Only $0.2 \%$ of the $\mathrm{Si}$ atoms are shown for visualization clarity.

probe data set is apparent at $\sim 3 \times 18 / \mathrm{cm}^{3}$. The integrated dose was $8.82 \times 10^{14} / \mathrm{cm}^{2}$ and $9.2 \times 10^{14} / \mathrm{cm}^{2}$ for SIMS and atom-probe analysis, respectively.

While the SIMS and atom-probe analysis compare favorably, distinct differences exist between the two techniques. The SIMS analysis was sensitive to As and Si ions. Atomprobe tomography is sensitive to any field-evaporated ion species with a mass-to-charge state ratio between 1 and 200 amu. The spot size for SIMS analysis is typically $50 \mu \mathrm{m}$ in diameter, with atomic concentrations averaged over this region. The field of view for atom-probe analysis is typically $50-100 \mathrm{~nm}$ in diameter, with precision location of individual atomic species in all three dimensions. This enables accurate analysis of site-specific nanostructures and distinguishes physical variations in dopant distribution. This discrimination is apparent in the variance in the 1D concentration collected by the atom probe shown by the error bars in Fig. 3 . While the SIMS profile is smooth, the atom-probe data demonstrate the natural counting-statistical variance, in this case approximately $32 \%$, in dopant atoms in the $z$ direction of the specimen.

The gate region of $\mathrm{Si} \mathrm{CMOS}$ transistors is where the switching control action takes place. Nanoelectronic Si transistor gate regions consist of a thin (several $\mathrm{nm}$ ) gate dielectric film deposited onto the $\mathrm{Si}$. The conductive gate contact is

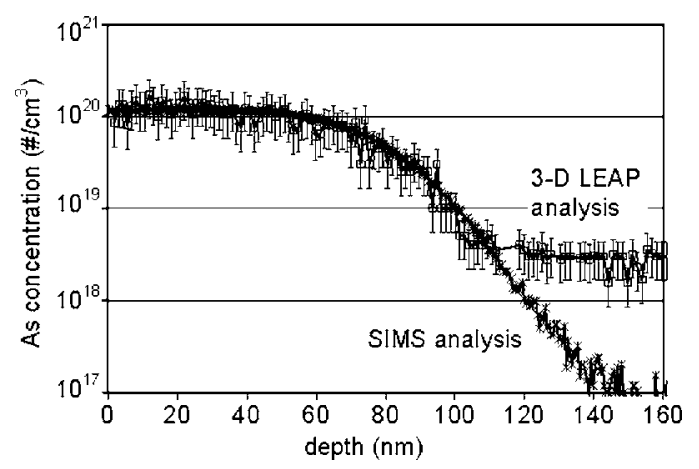

FIG. 3. A comparison of 1D As composition profiles obtained from 3D LEAP and SIMS analysis. LEAP and SIMS analysis.
Downloaded 25 May 2007 to 128.104.198.120. Redistribution subject to AIP license or copyright, see http://apl.aip.org/apl/copyright.jsp

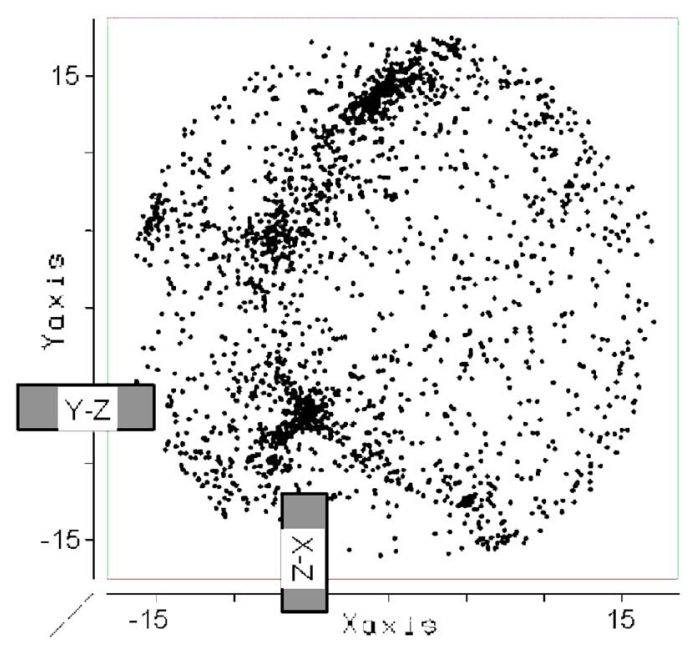

FIG. 4. A 3D atomic map showing the boron (black spheres) distribution in a heavily doped poly-Si structure. For clarity, the Si atoms are not shown. The cluster under investigation is in the bottom left corner and is demarked by the lateral $1 \mathrm{D}$ composition profile markers.

then fashioned by depositing a polycrystalline silicon (poly$\mathrm{Si}$ ) film on top of the gate dielectric, and heavily doping the poly-Si with ${ }^{11} \mathrm{~B}$. To increase its conductivity, the poly-Si is typically doped well beyond the dopant solubility limit; this results in a large concentration of inactive dopants in order to maximize the number of electrically active dopant atoms. The wafer is then heated to activate the dopants and to achieve a uniform dopant distribution throughout the polySi. Historically, the uniformity of the dopant distribution was measured using SIMS analysis. This analysis provides dopant profiling in the depth or " $z$ " direction only, with no information regarding the dopant distribution in the lateral or " $x-y$ " dimensions. Poly-Si layers, however, necessarily possess a grain structure, and preferential dopant diffusion to and along the grain boundaries, instead of diffusion into the grain interiors, is a reasonable expectation. Furthermore, highly overdoping a material often results in the formation of dopant clusters.

Figure 4 shows a plan-view image of a heavily doped poly-Si gate structure. In this figure, B atoms are shown as black spheres and the Si atoms are not shown. It is immediately clear that the dopant atoms are not uniformly distributed. The data imply that the B has segregated to the grain boundaries, decorating them and making them easily recognizable. Clustering clearly occurs, with a large dopant cluster evident where three grain boundaries meet at the bottom left section of Fig. 4. The existence of clustering at the intersection of grain boundaries implies that rapid diffusion of dopants down grain boundaries leads to systematic cluster formation at the intersection of multiple grains. A second possibility is that dopants diffuse through the bulk of the grain and segregate at the intersection of grains independent of grain-boundary diffusion. The calculated root-meansquared diffusion of $\mathrm{B}$ in poly-si $(\sqrt{6 D t}$ where $D$ is diffusivity and $t$ is time) for this example is approximately $22 \mathrm{~nm}$. Because this is on the order of the average grain size of 25 $\mathrm{nm}$, it is not possible to decisively select one mechanism over the other. In any event, this represents a potential challenge for the utilization of heavily doped poly-Si material in ever-smaller gate structures. Regardless of the uniformity of 

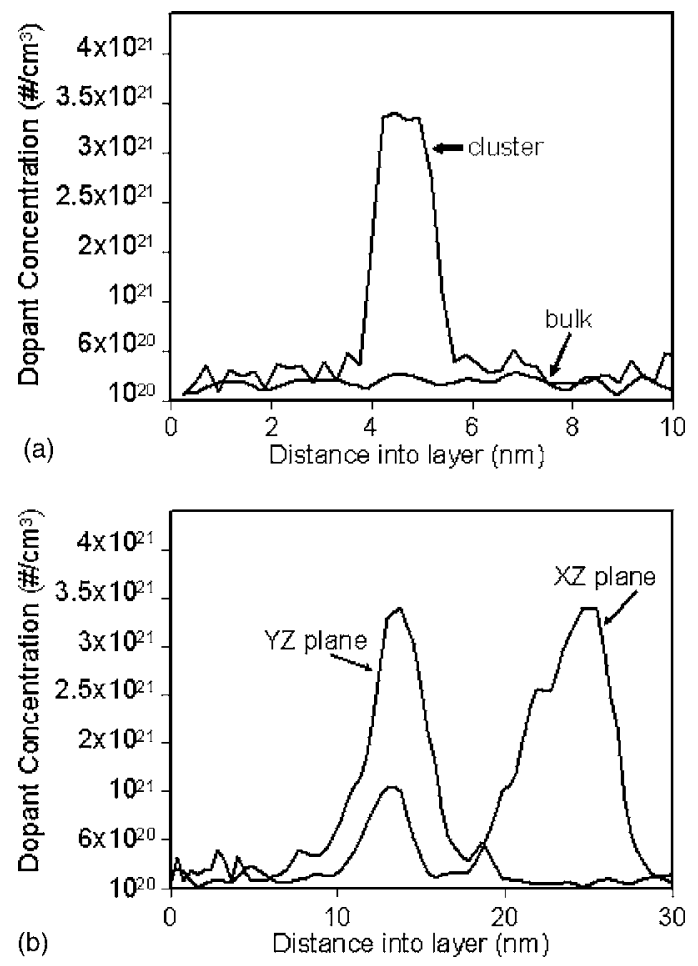

FIG. 5. (a) Longitudinal 1D composition profiles in the "z-direction" for the (a) bulk-Si region and for the cluster region. (b) Lateral $1 \mathrm{D}$ composition profiles through the cluster region along the (c) $X-Z$ and (d) $Y-Z$ planes.

most certainly results in large accumulations of dopants at the intersection of multiple grains.

To further examine the dopant cluster, 1D composition profiles were constructed from the 3D atomic map. These 1D profiles, Fig. 5, were created in all three directions of interest: (1) normal to the $X-Y$ plane, or in the " $z$ direction" to gain depth profiling; (2) as a chord normal to the $X-Z$ plane; and (3) as a chord normal to the $Y-Z$ direction. The $X-Z$ and $Y-Z$ chords are indicated in Fig. 4. The $Z$ profiling was done through the cluster region and in the bulk region of the largest grain.
The bulk profile shows a B concentration of $\sim 2$ $\times 10^{20} / \mathrm{cm}^{3}$ and excellent dopant uniformity through the depth of the poly-Si. In contrast, the 1D profiles through the cluster region, both longitudinal and lateral, demonstrate the localized nonuniformity in dopant distribution. The $z$-direction profile in the cluster region shows that the cluster is approximately $2 \mathrm{~nm}$ in depth, and the $1 \mathrm{D}$ profiles intersecting the cluster region parallel to the $X-Y$ plane show that the cluster is $\sim 8 \mathrm{~nm}$ and $\sim 7 \mathrm{~nm}$ long in the $Y-Z$ and $X$ $-Z$ planes, respectively. There are $264 \mathrm{~B}$ atoms captured within the cluster, amounting to an approximate dopant concentration of $3 \times 10^{21} / \mathrm{cm}^{3}$ in this volume. While the atom probe cannot determine the electrical activity of each dopant it is likely that the majority of dopants in the cluster are inactive.

The utilization of a local electrode has transformed the traditional 3D atom probe into an effective diagnostic for doped-Si nanostructures. An atom probe that utilizes a local electrode geometry is capable of analyzing large volumes of $\mathrm{Si}$ relative to the nanostructure of interest. The traditional dopant species are resolved and mapped independent of mass or electrical activity. This microscopy capability provides a powerful new tool to investigate Si nanodevices. It is anticipated that the continued application of 3D local electrode atom probe diagnostics for the study of $\mathrm{Si}$ devices on the nanoscale will accelerate the development of advanced nanoelectronic devices.

${ }^{1}$ P. M. Voyles, D. A. Muller, J. L. Grazul, P. H. Citrin, and H. J. L. Gossmann, Nature (London) 416, 826 (2002).

${ }^{2}$ P. M. Voyles, D. A. Muller, and E. J. Kirkland, Microsc. Microanal. 10, 291 (2004).

${ }^{3}$ F. Hillion, F. Horreard, and F. J. Stadermann, in Proceeding, of the $12^{\text {th }}$ International Conference on Secondary Ion Mass Spectrometry, edited by R. Lareau, G. Gillen, and S. Hues, Gaithersburg, MD, 1999, pp. 209-212.

${ }^{4}$ T. F. Kelly, T. T. Gribb, J. D. Olson, R. L. Martens, J. D. Shepard, S. A. Weiner, T. C. Kunicki, R. M. Ulfig, D. R. Lenz, E. M. Strennen, E. Oltman, J. H. Bunton, and D. R. Strait, Microsc. Microanal. 10, 373 (2004).

${ }^{5}$ T. F. Kelly and M. K. Miller, Rev. Sci. Instrum. (unpublished).

${ }^{6}$ D. J. Larson, D. R. Foord, A. K. Petford-Long, H. Liew, M. G. Blamire, A. Cerezo, G. D. W. Smith, Ultramicroscopy 79, 287 (1999). 